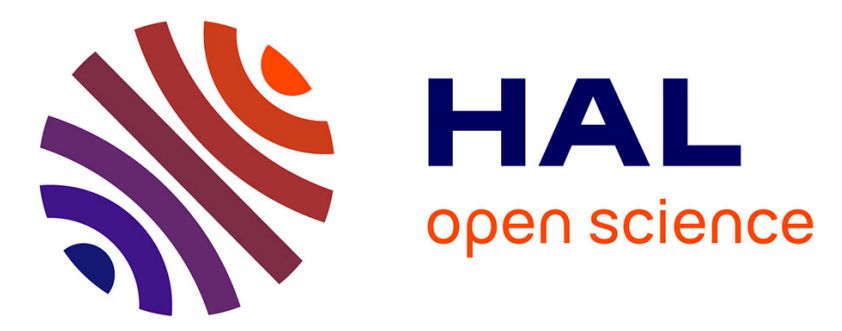

\title{
Enforcing consistency during the adaptation of a parallel component
}

\author{
Jérémy Buisson, Françoise André, Jean-Louis Pazat
}

\section{To cite this version:}

Jérémy Buisson, Françoise André, Jean-Louis Pazat. Enforcing consistency during the adaptation of a parallel component. The 4th International Symposium on Parallel and Distributed Computing (ISPDC), Jul 2005, Lille, France. pp.66, 10.1109/ISPDC.2005.24 . hal-00498839

\section{HAL Id: hal-00498839 \\ https://hal.science/hal-00498839}

Submitted on 8 Jul 2010

HAL is a multi-disciplinary open access archive for the deposit and dissemination of scientific research documents, whether they are published or not. The documents may come from teaching and research institutions in France or abroad, or from public or private research centers.
L'archive ouverte pluridisciplinaire HAL, est destinée au dépôt et à la diffusion de documents scientifiques de niveau recherche, publiés ou non, émanant des établissements d'enseignement et de recherche français ou étrangers, des laboratoires publics ou privés. 


\section{Enforcing consistency during the adaptation of a parallel component}

\author{
Jérémy Buisson \\ IRISA/INSA de Rennes \\ avenue du Général Leclerc \\ 35042 Rennes, France \\ Email: jeremy.buisson@irisa.fr
}

\author{
Françoise André \\ IRISA/Université de Rennes 1 \\ avenue du Général Leclerc \\ 35042 Rennes, France \\ Email: francoise.andre@irisa.fr
}

\author{
Jean-Louis Pazat \\ IRISA/INSA de Rennes \\ avenue du Général Leclerc \\ 35042 Rennes, France \\ Email: jean-louis.pazat@irisa.fr
}

\begin{abstract}
As Grid architectures provide execution environments that are distributed, parallel and dynamic, applications require to be not only parallel and distributed, but also able to adapt themselves to their execution environment. This article presents a model for designing self-adaptable parallel components that can be assembled to build applications for Grid. This model includes the definition of a consistency criterion for the dynamic adaptation of SPMD components. We propose a solution to implement this criterion. It has been evalued on both synthetic and real codes to exhibit the behavior of the several proposed strategies.
\end{abstract}

Keywords - dynamic adaptation, consistency, parallel computing

\section{INTRODUCTION}

Grid is an emerging architecture that we consider as a largescale federation of pooled resources. Those resources might be processing elements, storage, and so on; they may come from parallel machines, clusters, or any workstation. One of the main properties of these resources is to have changing characteristics even during the execution of an application. Resources may come and go; their capacities may vary. Moreover, resources may be allocated then reclaimed and reallocated as applications start and terminate on the Grid. To sum up, Grid is an architecture that is at the same time distributed, parallel and dynamic. This is why we think that the right application model to use in order to benefit from such an environment should be distributed assemblies of parallel self-adaptable components.

In our context, we just see the component as a unit that explicitly specifies the services it provides ("provide ports") and the ones it requires ("use ports"). Required resources are one kind of required services. Service specifications can be qualitative (type specification); they can also be quantitative (quality of the provided or used services). A parallel component is simply a component that encapsulates a parallel code. For example, GridCCM [1] extends CORBA Component Model to support parallel components. A self-adaptable component is a component that is able to modify its behavior depending on the changes of the environment: it may use different algorithms that use services differently and provide different qualities of services. The choice of the algorithm is a component-dependant problem and cannot be solved in a general way at some middleware level. Nevertheless, some generic mechanisms exist in adaptable components that are independant of the component itself. Our research focuses on the design of an adaptation framework for parallel components that provides all those common mechanisms. One of the main problems consists in providing a mechanism for choosing a point in the execution to perform the adaptation in a consistent manner. This article aims at addressing this problem.

Section II presents our model of dynamic adaptation for parallel codes to set the context of this article up. Section III gives few mathematical definitions that are useful in the remaining of this paper. Sections IV and V describe how the future of the execution path is predicted to choose the adaptation point at which the adaptation code is inserted. Section VI discusses the results obtained from our experiments. Section VII compares our work to others in the area of dynamic adaptation. It also shows similarities with computation steering and fault tolerance. Endly, section VIII concludes this paper and presents some perspectives.

\section{Model OF SELF-ADAPTABLE COMPONENTS}

The dynamic adaptability of a component is its ability to modify itself according to the execution environment on which it is deployed. The goal is to help the component to give the best performance given its allocated resources. Moreover, it makes the component aware of changes in resource allocation. Resources may be allocated and reclaimed dynamically during the lifetime of the component.

\section{A. Structure of a self-adaptable component}

With our model of dynamic adaptation [2], parallel selfadaptable components can split in several functional boxes (some of them being independant of the component itself). A parallel self-adaptable components contains five major functional boxes: its service, reactions, policy, decider and coordinator. Figure 1 shows the connections between those boxes. A short description of them follows.

- Service. The service of the component is what it provides. The service can be implemented by several algorithms that might be used to solve a single problem. Each of these algorithms uses differently the resources; thus, there is one preferred algorithm for each configuration of the resources allocated to the component. 


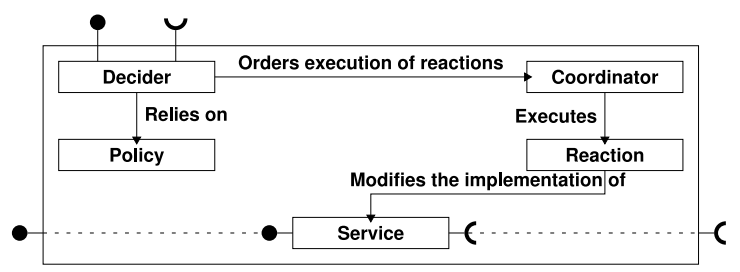

Fig. 1. Model of a parallel self-adaptable component

- Reactions. The reactions are the ways to modify the component. They modify the way the service is implemented, changing the algorithm with another one, adjusting its parameters or anything else.

- Policy. The policy describes the component-specific information required to make the decisions. For example, the policy can be an explicit set of rules or the collection of the performance models of the algorithms.

- Decider. The decider makes the decisions. It decides when and how the component should adapt itself. In order to decide, it relies on the policy ; it may also embed a feedback controller (for example to complement the performance models of the policy).

- Coordinator. The coordinator synchronizes the service with the reaction. To do so, it chooses a point (an instantaneous statement that annotates a special state) within the execution path in order to insert the reaction chosen by the decider.

Service, reactions and policy are specific to the component, whereas the decider and the coordinator are independant of the component. The decider and the coordinator should thus be parts of an adaptation framework.

In the context of parallel components, the service can be implemented either sequentially (exactly one execution thread) or a parallelly (several communicating execution threads). In the latter case, the coordinator must implement a parallel algorithm.

\section{B. Contracts between components}

Because the quality of the services used by a component is of greater importance to the adaptation, we attach to each port link a contract that describes the effective quality of the provided service. Moreover, for the sake of uniformity, our model abstracts resources as "provide ports" of some component called the environment. The execution environment of a component is thus completely described by the contracts attached to its "use ports". Consequently, any change in the execution environment is reflected by a change in those contracts, triggering the adaptation.

Contracts are dynamically negotiated and renegotiated: a component negotiates sufficient quality of service with its subcontractors in order to respect the contracts with its clients; it negotiates with its clients the quality of the provided services according to the ones contracted with its subcontractors. In that way, any renegotiation of one contract automatically propagates to the entire assembly of components (the application) to adjust the quality of all the provided services of all components. Consequently, it propagates the trigger of the adaptation to the components that require it.

\section{Performance model}

The goal of dynamic adaptation is to help the component to give the best performance. Thus, the model assumes that adaptation occurs when the component runs a non-optimal service given its allocated resources. This means that the completion time is worse than what it could be. In a general way, the conceptual performance model of a single-threaded component that adapts itself is shown by figure 2 . The $\mathrm{x}$-axis is the execution flow of the component; the curve gives the expected completion time if the component adapts itself at the corresponding point in its execution flow.

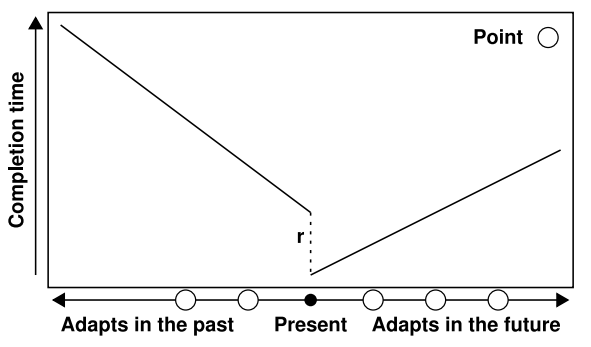

Fig. 2. Conceptual performance model of an adapting component

The adaptation can occur in either the future or the past of the current state. In the case of adapting in the past, it requires the restoration of a checkpoint (that has a cost $r$ on the curve). The limit of adapting in the future is adapting once the execution has ended (or equivalently not adapting at all); the limit of adapting in the past is to restart from the begining (purely static adaptation). It appears that whatever the search direction, the best point will always be the nearest one from the current state.

In the case of a parallel component, several curves are superimposed. Because the threads of the component are not necessarily synchronized, the curves are slightly shifted. The global completion time is thus the maximum of the ones for all threads. In this case, the best point can be in the future for some threads and in the past for the others.

In this paper, we arbitrarily chose to consider only the search direction that goes to the future of the execution. In the case of a parallel component, we look for a global point that is in the future of all threads.

\section{Consistency model}

In the case of a parallel service, the coordinator must enforce the consistency of the adaptation. We defined one consistency model we called the "same state" consistency model. In this model, the adaptation is said to be consistent if and only if all threads execute the reaction from the same state (this logical synchronization does not require an effective synchronization of the execution threads).

This model only makes sense in the case of SPMD codes (parallel codes for which all threads share the same control flow graph). 


\section{E. Scenario of dynamic adaptation}

From time to time, possibly due to some external event, the decider decides according to the policy that the component should adapt itself. It thus orders the coordinator to execute a specified reaction. The coordinator then chooses a point in the future of the execution path at which the reaction can be executed. When the service is parallel, the point is a global state such that all threads are in the same state ("same state" consistency model). At this point, the coordinator suspends the service and executes the reaction.

In this paper, we will concentrate on the coordinator. Section IV describes how the point can be computed; section V depicts how an algorithm can be built. In the remaining of the document, we will call "candidate points" the points at which a reaction can be executed. The one that has been chosen by the coordinator will be called the "adaptation point".

\section{DEFINITIONS}

Definition 1 (poset): A poset (or partially-ordered set) is a pair $(P, R)$ where $P$ is a set and $R$ is a binary relation over $P$ that is (1) reflexive, (2) antisymmetric and (3) transitive.

(1) $\forall x \cdot(x \in P \Rightarrow x R x)$

(2) $\forall x, y \cdot\left(x, y \in P^{2} \Rightarrow((x R y \wedge y R x) \Rightarrow x=y)\right)$

(3) $\forall x, y, z \cdot\left(x, y, z \in P^{3} \Rightarrow((x R y \wedge y R z) \Rightarrow x R z)\right)$

Definition 2 (supremum): Given a poset $(P, R)$, the supremum $\sup (S)$ of any subset $S \subset P$ of $P$ is the least upper bound $u$ of $S$ in $P$ such that:

$$
\begin{aligned}
& \forall x \cdot(x \in S \Rightarrow x R u) \\
& \wedge \forall v \cdot(v \in P \Rightarrow((\forall x \cdot(x \in S \Rightarrow x R v)) \Rightarrow u R v))
\end{aligned}
$$

Definition 3 (infimum): Symmetrically to the supremum, given a poset $(P, R)$, the infimum $\inf (S)$ of any subset $S \subset P$ of $P$ is the greater lower bound $l$ of $S$ in $P$ such that:

$$
\begin{aligned}
& \forall x \cdot(x \in S \Rightarrow l R x) \\
& \wedge \forall v \cdot(v \in P \Rightarrow(\forall x \cdot(x \in S \Rightarrow v R x)) \Rightarrow v R l)
\end{aligned}
$$

The infimum of $S$ in the poset $(P, R)$ is the supremum of $S$ in $\left(P, R^{-1}\right)$.

Definition 4 (lattice): A lattice $\mathcal{L}$ is a poset $(P, R)$ such that for any pair $x, y \in P^{2}$, both the supremum $\sup (\{x, y\})$ and the infimum inf $(\{x, y\})$ exist. This defines two internal composition laws:

$$
\begin{aligned}
& \sup (\{x, y\})=x \vee y \quad \text { (join) } \\
& \inf (\{x, y\})=x \wedge y \quad \text { (meet) }
\end{aligned}
$$

\section{COMPUTING THE FUTURE}

The coordinator needs to find a global point such that it represents the same state for all threads. As it restricts to points in the future of execution paths this point should be the first one in the future for all threads, given the assumption on the performance model.

\section{A. Local prediction of the next point}

For each thread, the coordinator needs to find the next candidate point in the future of the execution path. This point would be the one at which the coordinator is going to execute the reaction in the case of a single-threaded component.
1) General schema: An execution path can be seen as a path in the control flow graph that models the code. If loops are unrolled (for example by tagging nodes with indices in iteration spaces), the control flow graph defines a "precedes" partial order between the points of a code. Given this relation, each thread of the parallel component is able to locally predict its next point at least in trivial cases.

2) Uncertain predictions: Predicting the future of an execution path is undecidable due to conditional instructions: their behavior is unpredictable since it depends on runtime computations. This occurs for both conditions and loops, which appear as nodes having several successors in the control flow graph.

Three strategies may be used when such a node is encountered during the computation of the adaptation point.

- Postpone. The computation of the adaptation point can be postponed until the effective behavior of the instruction is known. Once the unpredictable instruction has been executed, the computation of the adaptation point resumes with a new start point (the one that is being entered).

- Skip. The computation of the point can move forward in the control flow graph until all control subflows merge. In this case, the prediction jumps over loops and conditions.

- Force. The behavior of the conditional instruction can be guessed when additional static information is available. With this strategy, one branch of the conditional instruction is assumed accordingly to some applicationdependant knowledge; then some application-level mechanism enforces that the execution path respects this assumption.

The two following examples illustrate the usage of the "force" strategy. Firstly, it is possible for some loops to insert unexpected empty iterations; in this case, it can be guessed that the conditional instruction will execute the branch that stay in the loop; this behavior can be enforced by making one more empty iteration on-demand. Secondly, if the code includes assertions to detect error cases, it could be reasonably assumed that those assertions hold; in this case, it can be guessed that the corresponding conditional instruction will execute the branch leading to error-free cases.

\section{B. Characterization of the chosen global point}

The point that should be chosen is the supremum $u$, if it exists, of the set $S$ of the next local points for each thread with respect to the "precedes" partial order (noted $\preceq$ ). Indeed, the property $\forall x \cdot(x \in S \Rightarrow x \preceq u$ ) guarantees that $u$ is in the future of all threads; the property $\forall v$. $(v \in P \Rightarrow((\forall x \cdot(x \in S \Rightarrow x \preceq v)) \Rightarrow u \preceq v))$ means that $u$ is the first point amongst those in the future of all threads.

Even if loops are unrolled, the control flow graph is not a lattice as it may contain patterns such as the one of figure 3(a). In this example, the supremum sup $(\{P 1, P 2\})$ does not exist. This pattern captures a subset of conditional instructions. The three strategies that has been identified for locally predicting the next point can be used to work-around non-existence of the supremum. 


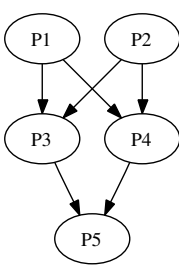

(a) Pattern which prevents the supremum from existing

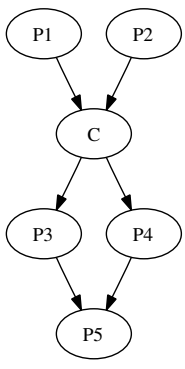

(b) Inserting nodes for conditionals

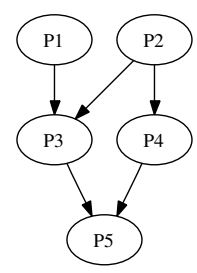

(c) Removing branches of conditionals
Fig. 3. Example of control flow graph with non-existent supremum

- Postpone. Postponing the computation of the adaptation point until the unpredictable instruction has been executed can be seen as inserting a special node that models that instruction in the control flow graph. For example, on figure 3(b), a node $C$ has been inserted to represent the conditional instruction. When such a node is chosen, a new round for finding a satisfying point is started once the corresponding instruction has been executed.

- Skip. Skiping the conditional block is equivalent to compute the supremum within the subset defined by:

$\{a \mid \forall y \cdot((\forall x \cdot(x \in S \Rightarrow x \preceq y)) \Rightarrow(a \preceq y) \vee(y \preceq a))\}$

This set contains the points that are related to all successors of all points in $S$. Those points are guaranteed to be traversed by any execution thread starting from any point in $S$. In the given example, it restricts to compute the supremum $\sup (\{P 1, P 2\})$ in $\{P 1, P 2, P 5\}$; this supremum exists.

- Force. Forcing one branch of a conditional instruction removes all out edges but one of the corresponding node. This is sufficient to make the supremum exist. For example, on figure 3(c), the conditional instruction that follows $P 1$ has been forced such that it always leads to P3.

Each conditional instruction may use a distinct strategy. In such a case, the several strategies are combined.

\section{Comparison of the strategies}

These three strategies have their own fall down. The "postpone" one shortens the time between choosing a point and reaching the chosen point. It may result in an increase of the risk that the execution thread tries to get through the locally chosen point before it has been globally either confirmed or evicted. The "skip" one chooses an adaptation point further in the future of the execution path (and possibly falls back on the end of the code). The "force" one modifies the code of the execution thread.

\section{BUILDING AN ALGORITHM}

In order to solve the problem of finding a point in the case of a parallel service, an algorithm has been designed.

\section{A. Identification of the candidate points}

Having a good identification system for the points is a key issue. Indeed, as section IV-B showed, the case of parallel self-adaptable components requires the computation of the supremum of a set of points. This requires to be able to compare easily candidate points with respect to the "precedes" partial order. However, with naive point identification, deciding whether a candidate point precedes, succeeds, or is not related to another one requires the computation of the transitive closure of the control flow graph. The same apply for the join composition law. This is why smarter identification system for the points is needed.

1) Description of the identification system: We think that the good representation to use is a tree view of the hierarchical task graph. Figure 4 gives an example algorithm that has been annotated with candidate points; figure 5 shows the control flow graph between annotated points; figure 6 shows the corresponding tree view. The edges of the tree are labelled as follows:

- out edges of loop nodes are labelled with the value of the indice within the iteration space of the loop;

- out edges of condition nodes are labelled with either the symbol "then" (the condition is true) or the symbol "else" (the condition is false);

- other edges (out edges of block nodes) are labelled with the execution order number in the control flow.

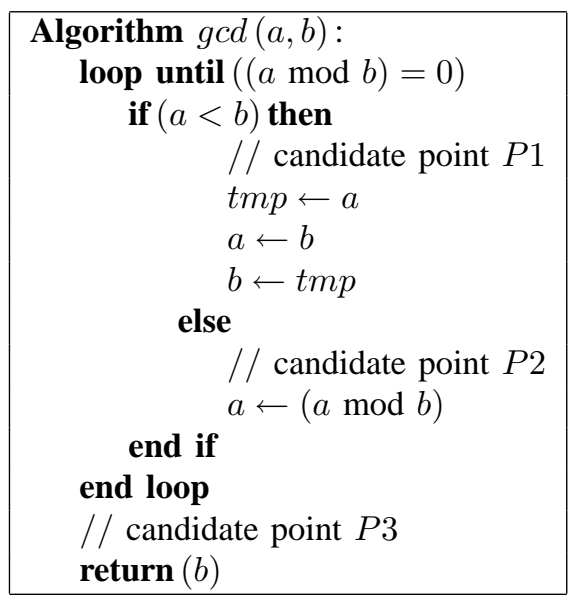

Fig. 4. An example algorithm

Considering the tree view of the hierarchical task graph, one can see that the nodes of the control flow graph are the leaves of the tree. Each candidate point is identified by the sequence composed of the labels of the edges traversed by the path from the root to the node corresponding to it. For example, the node " $P 3$ "is identified by $\langle 2\rangle$; the node " $P 1$ " is identified by the sequence $\langle 1,(a, b), 1$, else, 1$\rangle$. 


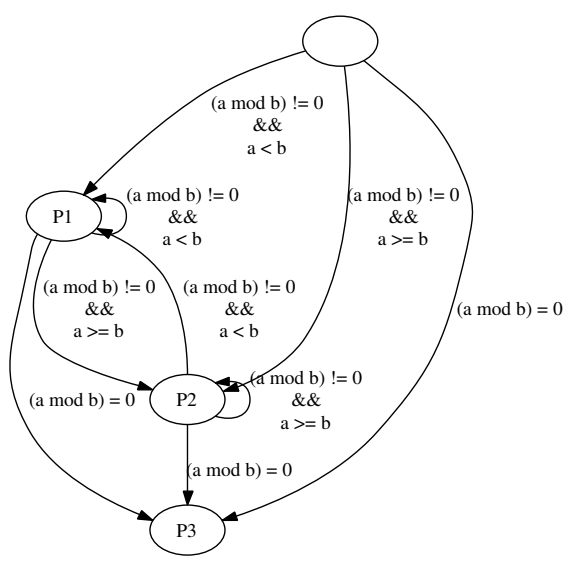

Fig. 5. Control flow graph of the algorithm 4

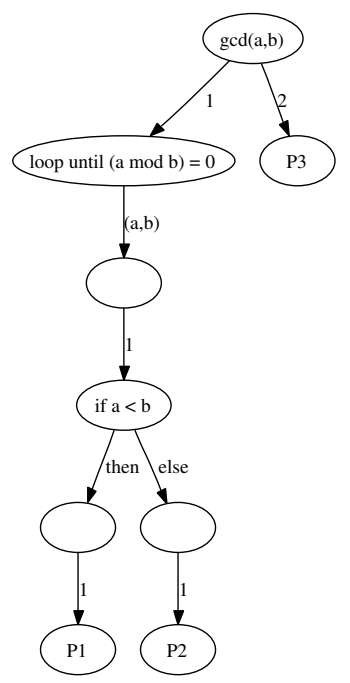

Fig. 6. Tree view of the hierarchical task graph of the algorithm 4

2) Order on points: The set of edge labels in the tree is $\mathcal{E}=\mathbb{N} \cup \mathcal{I} \cup\{$ then, else $\}$, where $\mathbb{N}$ is the set of execution order numbers (natural numbers) and $\mathcal{I}$ the iteration space partially ordered by an application-specific relation $\preceq_{\mathcal{I}}$. We define the following partial order over the set $\mathcal{E}$ of edge labels of the tree:

$$
\begin{aligned}
\forall x, y \cdot\left(x, y \in \mathcal{E}^{2}\right. & \Rightarrow \\
(x \preceq \mathcal{E} y \Leftrightarrow & \left(\left(x, y \in \mathbb{N}^{2} \wedge x \leq y\right)\right. \\
& \left.\left.\left.\vee\left(x, y \in \mathcal{I}^{2} \wedge x \preceq \mathcal{I} y\right)\right)\right)\right)
\end{aligned}
$$

Notably, the two constants "then" and "else" are set not comparable to other values and values in iteration spaces (such as $(a, b)$ in the example) are partially ordered by an application-specific relation.

The edges of the control flow graph (that represent the "precedes" partial order between points) are (graphically) transverse to those of the tree. Consequently, the lexicographical partial order $\preceq_{\mathcal{E}}^{n}$ on the sequences is equivalent to the partial order on points defined by the control flow graph. Thus, with this identification system, deciding whether a point precedes, succeeds, or is not related to another one can be computed as a direct lexicographical comparison (not requiring the computation of the transitive closure of the control flow graph anymore). Moreover, this ordering directly takes into account the loop indices in iteration spaces, whereas the control flow graph does not.

3) Discussion: This identification system makes sense only in the case of a parallel component into which all threads are allowed to have different dynamic behaviors (different behavior of conditionnal instructions and loop indices desynchronization). Otherwise, a simple counting identification system is sufficient. Furthermore, in the case of a non-parallel component, no identification system is required.

\section{B. Computation of the local adaptation point}

Because we restricted to candidate points in the future of the execution path, the local adaptation point is the next point in the path. Computing this point simply consists in following the edges of the control flow graph. This corresponds to a depth-first traversal of the tree from the left to the right. This traversal begins at a start point that is at least the one being executed at the time of the computation.

\section{Instrumentation of the code}

In order to predict the next candidate point in the future of the execution path, the coordinator must be able to locate the actual execution progress in the space of the candidate points. As it must be able to build the sequence identifying the candidate point that is being executed by the execution thread, the coordinator requires an instrumentation of the code.

The instrumentation of the code consists in inserting some pieces of code at each node of the tree view of the hierarchical task graph as presented at the preceding section. The figure 7 shows an example of an instrumented algorithm.

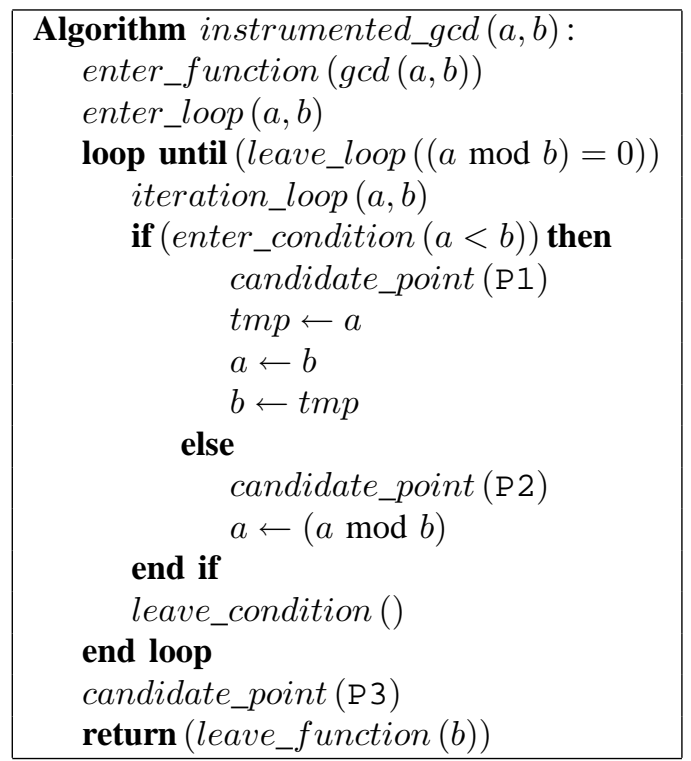

Fig. 7. Instrumented version of the algorithm 4

The process of inserting the instrumentation statements in the source code of the component can be largely automated 
using aspect-oriented programming (AOP [3]) techniques. In this case, the join points are the nodes of the tree representation of the code previously described.

\section{Protocol for computing the supremum}

Many trivial protocols might be designed to compute this supremum (for example, centralized). Many already exist to compute a maximum (in particular for leader election); they can easily be modified to compute a supremum instead of a maximum. However, we think that a specific protocol suits better this case.

1) Motivation for designing a specific protocol: The full set of the local adaptation points is not always necessary to compute the supremum. The idea is to superimpose the agreement protocol and the local computations of the next candidate point in order to exempt some of the execution threads from computing their local adaptation point. In particular, it is interesting to exempt those whose prediction is postponed due to conditional instructions. Indeed, it allows the computation of the local adaptation point to resume sooner with a start point that is further in the execution path. Moreover, superimposing the protocol and the computation evicts the earliest adaptation points sooner than if the whole set of local adaptation points must be computed. This avoids situations in which the execution thread tries to execute through a locally chosen point that has not been either confirmed or evicted yet.

2) Informal description of the protocol: The key idea of the protocol is to let the threads negotiate. Each thread can propose to the others its view of what the common adaptation point is, namely the point it has chosen locally. If it has not chosen one (because of unpredictable instructions such as conditions), it can give clues to the other threads. These clues can be for example the root node of the subtree representing the conditional block. When a thread receives a proposition or a clue, it may take different actions depending on how it compares to its own proposition.

- If its own proposition is better (that is to say in the future of the received one), it rejects the received proposition.

- If the received one is better, it is adopted.

- If both are not comparable, the thread retracts its proposition and computes a new one that takes into account the two previous ones. The computation starts at the point at which the control subflows merge (the supremum of the two points).

- If both propositions are the same, the thread agrees with the other one.

The protocol also progresses when an execution thread provides new information. For example when the functional thread executes a conditional instruction, it may retract its clue and propose a new one.

The protocol ends when all threads agree with each other's and when the agreement is on a true proposition (not just a clue). This means that they all have adopted the same point.

3) Anatomy of the protocol: We have designed a specific protocol to solve this problem. This protocol is based on a unidirectional ring communication scheme. Each thread confronts its proposition with the one of its predecessor and sends it only to its successor in the ring. The end of the protocol, namely the reach of the agreement, is detected using a Dijkstra [4]-like termination algorithm. Our protocol distinguishes strong propositions (values that can be chosen by the agreement) from weak propositions (values that forbid the agreement to conclude, previously called clues). The protocol allows threads to retract their proposition. It strictly defines the criteria of the consistency of retraction.

Our protocol is pessimistic. If an execution thread tries to get through a point thought to be the chosen one, the algorithm refuses to speculate whether it should allow the execution thread to continue or execute the reaction. It rather suspends the execution thread until it gets certainty. As a result, because the "postpone" strategy eases such situations (as described in section IV-C), this strategy tends to increase the risk of suspending the execution thread.

\section{EXPERIMENTS}

The experiments presented here aim at comparing the strategies described at section IV-A.2 with regard to functional code suspension and delay before the execution of the reaction. The goal is to exhibit and validate the expected behaviors described in section IV-C. This characterization would help developers to choose the right strategy depending on their code.

Three experiments has been made: synthetic loop code, synthetic condition code and NAS Parallel Benchmark 3.1 [5] FFT code. Only the results of the latter will be presented and discussed.

\section{A. Experimental protocol}

The experimental protocol consists in trigerring adaptation many times at random intervals (with uniform distribution to avoid implicit synchronization between the functional code and the adaptation trigger) while the code is being executed by a 4 processors cluster. Experiments result in two data series: one indicates for each adaptation the time elapsed between the trigger and the effective execution of the reaction; the other gives the time during which the functional thread has been suspended while choosing the point at which to adapt. For each data serie, one curve is drawn that gives an approximate measure of the density of samples for each observed value.

\section{B. Observations}

Figures 8 and 9 show the results with the FFT code. This experiment aims at comparing the "postpone" and "skip" strategies for conditional instructions within a main loop. The main loop has the "force" strategy so that the synchronization and communication statements induced by our algorithm are exclusively related to the inner instructions. This prevents distortions of the observations.

Figure 8 shows that the "postpone" strategy tends to select an adaptation point that arrives sooner in the execution path than the "skip" strategy. Conversly, figure 9 shows that the "postpone" strategy tends to suspend the functional code for a longer time than the "skip" strategy. Indeed, the "postpone" 


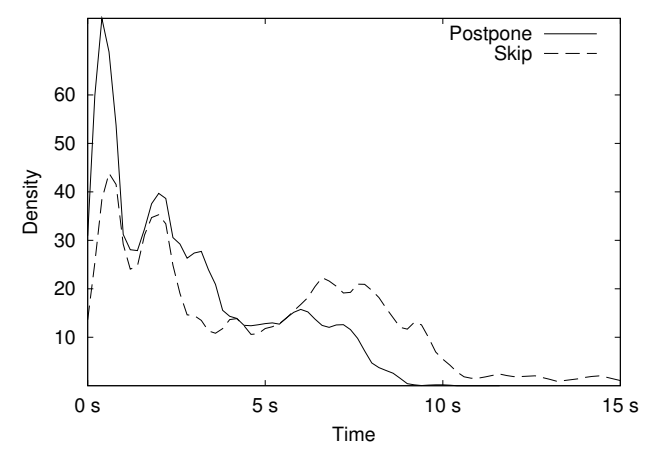

Fig. 8. Density curve for adaptation delay

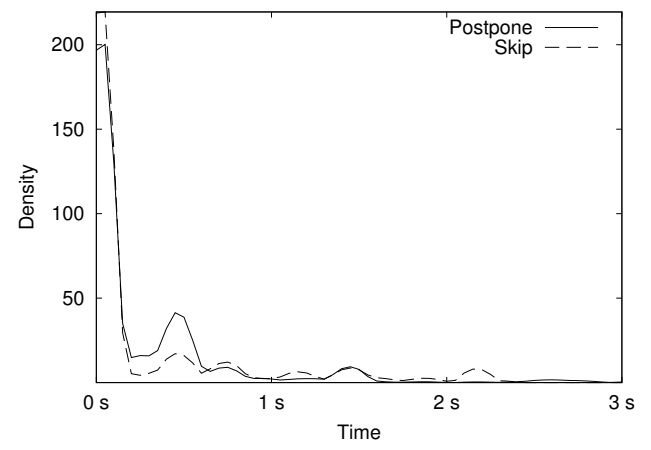

Fig. 9. Density curve for suspension time

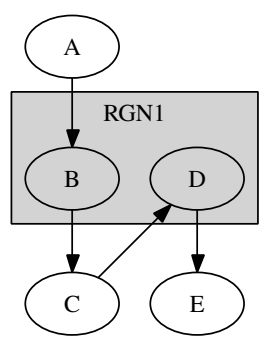

Fig. 10. Example of a region in a PCL static task graph strategy leads to higher density of samples up to about $0.5 \mathrm{~s}$ of functional code suspension. However, this observation has to be balanced as it appears that both strategies do not suspend the functional code in most of the samples.

\section{Discussion}

These results and those we obtained with synthetic codes confirm the behaviors we expected at section IV-C. Namely, the choice between the "postpone" and the "skip" strategies, is a trade-off between the precision of the prediction and the risk to uselessly suspend the functional code.

The observations are explained by the fact that the "postpone" strategy tends to delay the agreement in order to choose a more precise point (closer to the current state), in comparision to the "skip" strategy. It makes the "postpone" strategy increase the risk for other threads to reach that point before the agreement is made, and thus to suspend their execution. On the other side, the "skip" strategy tends to choose a point that is further in the future of the execution path; it results in a lower risk for other threads to reach that point before the agreement is made and thus to suspend their execution.

Consequently, if the time between a conditional instruction and the following point is large enough to balance the delay of the agreement, the "postpone" strategy should be preferred as it results in a sooner adaptation. Otherwise, the "skip" strategy should be used in order to avoid the suspension of the execution thread.

\section{RELATED WORKS AND DOMAINS}

\section{A. Other projects about dynamic adaptation of parallel codes}

Most of the projects that faced the problem of dynamic adaptation such as DART [6] do not consider parallelism. Other projects such as SaNS [7] and GrADSolve [8] build adaptive components for Grid. Those projects target adaptation only at the time of component invocation. This supposes a fine-grain encapsulation in components; otherwise, if methods are too long, adaptation is defeated because it cannot occur during an execution. Within the context of CommercialOff-The-Shelf (COTS) component concept, sufficiently finegrained encapsulation for the dynamic adaptation to make sense only at invocation level does not appear valuable enough; whereas sufficiently coarse-grained encapsulation for valuable COTS components are not able to really dynamically adapt themselves if they do so only at each invocation. This is why we think that components should be able to adapt themselves not only at method calls, but also during the execution of methods.

The Grid.It project [9] also faces the problem of dynamically adapting parallel components. The followed approach is based on structured parallelism provided by high-level languages. The compiler generates code for virtual processes that are mapped to processing elements at run-time. An adaptation consists in dynamically modifying this mapping. This adaptation can be done within language constructs transparently to the developper. In this case, the consistency enforcement mechanism can rely on assumptions on the overall structure of the program as it is generated by a compiler.

PCL [10] focuses on the reflexive programming tools for the dynamic adaptation of parallel applications. This project considers dynamic adaptation as modifications on a static task graph modeling the code and provides at runtime primitive operators on that graph. It gives a full framework for reflexive programming that is useful to implement reactions. The problem of the coordination of the reaction and the execution threads of the functional code has been studied with PCL in [11]. Their approach to the problem of the coordination is similar to ours: the adaptation is scheduled to be executed at an adaptation point in the future of the execution path. However, the problem of dealing with unpredictable conditional instructions is not addressed. Furthermore, the consistency model is different from ours. In PCL, each reaction is triggered at any point of the specified region. For example, on figure 10, tasks $B$ and $D$ are not distinguished. According to the consistency model, in a two-threads component, if one is executing $A$ and the other $C$, a "region in" reaction targeting region $R G N 1$ is scheduled at the $A \rightarrow B$ edge for the first thread and at the $C \rightarrow D$ edge for the second one. We think that it is a better consistency model to schedule the reaction at the $C \rightarrow D$ edge for both threads (our "same state" consistency). One solution might be to put $B$ and $D$ in two different regions; but in this case, PCL fails specifying that the reaction can be scheduled either before $B$ or before $D$, but at the same edge for all threads. Furthermore, PCL is unable to handle the 
case of thread desynchronization in loops (one thread doing more iterations than another does) for regions being in such a loop. The simple region counter is not sufficient to solve this problem.

\section{B. Computation steering}

The problem of steering a parallel component is very close to our problem of adapting this component. Indeed, in both cases, we need to insert dynamically code at some global state. The EPSN project [12] aims at building a platform for online steering and visualization of numerical simulations. In [13], the authors describe the infrastructure they built to do the steering. Their structured dates is similar to our candidate point identification system. Both systems are based on similar representations of the code. Moreover, steering like dynamic adaptation tries to find the next candidate point in the future of the execution path. Thus, computation steering and dynamic adaptation may benefit from fusionning in a single framework.

\section{Fault tolerance}

Fault tolerance might be seen as a particular case of dynamic adaptation. Indeed, it is the adaptation to the "crash" of some of the allocated resources. However, things are not that simple. Fault tolerance encompasses two different problems: the recovery of the fault and the adaptation to the new situation. Only the second one really belongs to the problem of dynamic adaptation. Many strategies can be used to recover from faults. The closest one from dynamic adaptation is probably checkpointing in the sense it requires some global points. Whereas dynamic adaptation can look for a point in the future of the execution path, checkpointing requires a point in the past. Nevertheless, mechanisms required by dynamic adaptation may be useful to implement recovery strategies based on checkpointing. The instrumentation of the code can be used as hooks at which checkpoints can be taken. Indeed, it implicitly statically coordinates the checkpoints of all threads, avoiding the problem of computing a global consistent state. Reciprocally, dynamic adaptation can take advantage of the techniques that have been developped to checkpoint and restart computations in the area of fault-tolerance.

\section{CONLUSION}

In this paper, we have briefly described the overall model we introduced to build self-adaptable parallel components. This paper essentially focuses on the coordinator functional box. This coordinator is responsible for scheduling the reaction in the execution path of the several execution threads.

The protocol we designed follows an pessimistic approach with regard to the fact that the execution thread may go through a confirmed point. In the future of our work, we will study how optimistic approaches can be designed. For example, when the functional code reaches a point suspected to be chosen but not yet confirmed, it may continue its execution instead of waiting for the point to be either confirmed or evicted. If at the end that point is confirmed, the situation may be repaired by rolling-back the functional code.
Moreover, our coordinator searches adaptation points exclusively in the future of the execution path. However, this is an arbitrary choice that has no other justification than simplifying algorithms. In the future, it would search in both directions (future and past) to find the best adaptation point. We will study how algorithms can be generalized.

Endly, our "same state" consistency model has to be extended to the case of non-SPMD components: we have already thought of another consistency model defined by a customizable correspondence relation between the candidate points of the threads.

As a longer term research, we are working on completing our architecture in order to build a platform. In particular, we are investigating protocols for adaptation within assemblies of components. We are also studying how a resource manager could take advantage of applications able to adapt themselves.

\section{REFERENCES}

[1] C. Pérez, T. Priol, and A. Ribes, "A parallel corba component model for numerical code coupling," in Proc. 3rd International Workshop on Grid Computing, ser. Lect. Notes in Comp. Science, M. Parashar, Ed., no. 2536. Baltimore, Maryland, USA: Springer-Verlag, Nov. 2002, pp. 88-99, held in conjunction with SuperComputing 2002 (SC '02). [Online]. Available: http://www.irisa.fr/paris/Biblio/Papers/Perez/ PerPriRib02GC.ps

[2] F. André, J. Buisson, and J.-L. Pazat, "Dynamic adaptation of parallel codes: toward self-adaptable components for the grid," in Workshop on Component Models and Systems for Grid Applications (Held in conjunction with ICS'04), June 2004. [Online]. Available: http: //www.irisa.fr/paris/Biblio/Papers/Buisson/AndBuiPaz04CMSGA.pdf

[3] G. Kiczales, J. Lamping, A. Mendhekar, C. Maeda, C. Lopes, J.-M. Loingtier, and J. Irwin, "Aspect-oriented programming," in Proceedins of European Conference on Object-Oriented Programming, M. Akşit and S. Matsuoka, Eds., vol. 1241. Berlin, Heidelberg and New-York: Springer-Verlag, 1997 , pp. 220-242.

[4] E. W. Dijkstra, W.H.J.Feijen, and A. van Gasteren, "Derivation of a termination detection algorithm for distributed computations," 1982, published as [14]. [Online]. Available: http://www.cs.utexas.edu/users/ EWD/ewd08xx/EWD840.PDF

[5] NAS, "Parallel benchmark," http://www.nas.nasa.gov/Software/NPB/.

[6] P.-G. Raverdy, H. L. V. Gong, and R. Lea, "DART : a reflective middleware for adaptive applications," in OOPSLA'98 Workshop \#13 : Reflective programming in C++ and Java, Oct. 1998.

[7] J. Dongarra and V. Eijkhout, "Self-adapting numerical software for next generation application," Aug. 2002

[8] S. Vadhiyar and J. Dongarra, "GrADSolve: RPC for high performance computing on the grid," in Euro-Par 2003: Parallel Processing, vol. 2790, Aug. 2003.

[9] M. Aldinucci, S. Campa, M. Coppola, M. Danelutto, D. Laforenza, D. Puppin, L. Scarponi, M. Vanneschi, and C. Zoccolo, "Components for high performance grid programming in the grid.it project," in Workshop on Component Models andd Systems for Grid Applications, June 2004

[10] V. Adve, V. V. Lam, and B. Ensink, "Language and compiler support for adaptive distributed applications," in ACM SIGPLAN Workshop on Optimization of Middleware and Distributed Systems (OM 2001), Snowbird, Utah, June 2001

[11] B. Ensink and V. Adve, "Coordinating adaptations in distributed systems," in 24th International Conference on Distributed Computing Systems, Mar. 2004, pp. 446-455.

[12] "Epsn project." [Online]. Available: http://www.labri.fr/Recherche/ PARADIS/epsn/

[13] A. Esnard, M. Dussere, and O. Coulaud, "A time-coherent model for the steering of parallel simulations," in Europar 2004, Sept. 2004. [Online]. Available: http://www.labri.fr/Recherche/PARADIS/epsn/doc/ presentation_europar_2004.pdf

[14] E. W. Dijkstra, W.H.J.Feijen, and A. van Gasteren, "Derivation of a termination detection algorithm for distributed computations," Information Processing Letters, vol. 16, pp. 217-219, 1983. 